\title{
Radius of Robust Feasibility Formulas for Classes of Convex Programs with Uncertain Polynomial Constraints
}

\author{
M.A. Goberna, ${ }^{* \dagger}$ V. Jeyakumar, ${ }^{\ddagger}$ G. $\operatorname{Li}^{\S}$ and N. $\operatorname{Linh}^{\S}$
}

Final Revised Version: November 21, 2015

\begin{abstract}
The radius of robust feasibility of a convex program with uncertain constraints gives a value for the maximal 'size' of an uncertainty set under which robust feasibility can be guaranteed. This paper provides an upper bound for the radius for convex programs with uncertain convex polynomial constraints and exact formulas for convex programs with SOS-convex polynomial constraints (or convex quadratic constraints) under affine data uncertainty. These exact formulas allow the radius to be computed by commonly available software.
\end{abstract}

Keywords. Robust optimization. Convex programming. Data uncertainty. Radius of robust feasibility.

\section{Introduction}

Robust convex optimization [3, 4, 5, 11, 16, 17] deals with solutions of robust counterparts of uncertain convex programs where the data uncertainty is treated as deterministic, as opposed to stochastic that is used in stochastic programming. It has emerged as a powerful numerically tractable approach to treat uncertainty in convex programming. Yet, one notable limitation of its application is that the robust counterpart, where the uncertainty is enforced for every data within a specified uncertainty set, may not have a feasible solution, resulting in an infeasible robust convex program. A formula for calculating the maximal 'size' of the specified uncertainty set has long been sought so that feasibility of the robust convex program, known as robust feasibility, can be guaranteed.

In this paper, we provide such results by introducing the notion of radius of robust feasibility in robust convex optimization. It was inspired by the notion of consistency radius used in linear semi-infinite programming in order to guarantee the feasibility of the nominal problem under perturbations preserving the number of constraints [7, 8, 9]. This notion extends the concept of radius of robust feasibility introduced in 13 for robust linear programs.

We first derive an upper bound for the radius in the general case of convex programs with convex polynomial constraints under uncertainty both in the affine and non-affine data. We then present an exact formula for the radius of robust feasibility of a convex program with uncertain SOS-convex polynomial constraints under affine data uncertainty. In particular, we show that the radius of robust feasibility can be given in terms of the optimal value of a convex quadratic program with sum-of-squares constraints. This value can be found by solving an equivalently reformulated linear semi-definite programming (SDP in brief) problem. Thus, the radius can easily be calculated using commonly available algorithms and software. In the special case of convex programs with uncertain convex quadratic constraints under affine data uncertainty, we show that the radius of robust feasibility can be found by solving a simple explicit semi-definite linear program.

\footnotetext{
*Corresponding author. Tel.: +34 965903533. Fax: +34 965903531.

${ }^{\dagger}$ Dept. of Statistics and Operations Research, Alicante University, 03071 Alicante, Spain.

$\ddagger$ Dept. of Applied Mathematics, University of New South Wales, Sydney 2052, Australia.

E-mail addresses: mgoberna@ua.es (M.A. Goberna), v.jeyakumar@unsw.edu.au (V. Jeyakumar), g.li@unsw.edu.au (G. Li), honglinh98t1@yahoo.com (N. Linh).
} 
The paper is organized as follows. Section 2 provides an upper bound for the radius of robust feasibility for convex programs with uncertain convex polynomial constraints. Section 3 gives exact radius of robust feasibility formulas under affine data uncertainty for convex programs with SOS-convex polynomial constraints or convex quadratic constraints.

Notations: Before we move to the next section, we introduce some necessary notation. We denote by $0_{n}$ and $\|\cdot\|$ the vector of zeros and the Euclidean norm in $\mathbb{R}^{n}$, respectively. The inner product between $x \in \mathbb{R}^{n}$ and $y \in \mathbb{R}^{n}$, is defined by $\langle x, y\rangle=x^{T} y$. The closed unit ball and the distance associated to the above norm are denoted by $\mathbb{B}_{n}$ and $d$, respectively. Given $Z \subset \mathbb{R}^{n}$, int $Z, \operatorname{cl} Z$, bd $Z$, and conv $Z$ denote the interior, the closure, the boundary and the convex hull of $Z$, respectively, whereas cone $Z:=\mathbb{R}_{+}$conv $Z$ denotes the convex conical hull of $Z \cup\left\{0_{n}\right\}$. A symmetric $n \times n$ matrix $A$ is said to be positive semi-definite, denoted by $A \succeq 0$, whenever $x^{T} A x \geq 0$ for all $x \in \mathbb{R}^{n}$. We also use $S_{+}^{n}$ to denote the cone consisting of all symmetric $n \times n$ positive semi-definite matrices. The $(n \times n)$ identity matrix is denoted by $I_{n}$. Let $Z$ be a closed and convex set in $\mathbb{R}^{n}$ with $0_{n} \in \operatorname{int} Z$. We define a convex function $\phi_{Z}: \mathbb{R}^{n} \rightarrow \mathbb{R}_{+}$by $\phi_{Z}(x)=\inf \{t>0: x \in t Z\}$. The function $\phi_{Z}$ is indeed a positive homogeneous convex function and is known as the Minkowski functional in the convex analysis literature, and is an extension of the usual norm function. In particular, if $Z=\mathbb{B}_{n}$, then $\phi_{Z}(x)=\|x\|$.

\section{Uncertain Convex Polynomial Constraints}

We begin by examining a convex program with general uncertain convex polynomial constraints

$$
\min _{x \in \mathbb{R}^{n}}\left\{\bar{f}(x): \bar{g}_{j}(x) \leq 0, j \in J\right\},
$$

where $J=\{1,2, \ldots, q\}$ be a finite index set, $\bar{f}: \mathbb{R}^{n} \rightarrow \mathbb{R}$ is a convex function and $\bar{g}_{j}: \mathbb{R}^{n} \rightarrow \mathbb{R}$ is a convex polynomial. The robust counterpart of the uncertain convex program $(P)$ is given by

$$
\left(R P_{\bar{\alpha}}\right) \quad \min _{x \in \mathbb{R}^{n}}\left\{\bar{f}(x): \bar{g}_{j}(x)+\sum_{l=1}^{p} v_{j}^{l} \bar{g}_{j}^{l}(x)+a_{j}^{T} x+b_{j} \leq 0, \forall\left(v_{j},\left(a_{j}, b_{j}\right)\right) \in \bar{\alpha}_{j}\left(M \times \mathbb{B}_{n+1}\right), j \in J\right\},
$$

where $\bar{g}_{j}^{l}$ are convex polynomials on $\mathbb{R}^{n}, l=1, \ldots, p, v_{j}=\left(v_{j}^{1}, \ldots, v_{j}^{p}\right) \in \mathbb{R}^{p}, M \subset \mathbb{R}_{+}^{p}$ is a convex compact set with $0_{p} \in M$ and $\bar{\alpha}_{j} \geq 0, j \in J$. We assume throughout this section that

$$
\left\{x \in \mathbb{R}^{n}: \bar{g}_{j}(x)+\sum_{l=1}^{p} v_{j}^{l} \bar{g}_{j}^{l}(x)+a_{j}^{T} x+b_{j} \leq 0, \forall\left(v_{j},\left(a_{j}, b_{j}\right)\right) \in \bar{\alpha}_{j}\left(M \times \mathbb{B}_{n+1}\right), j \in J\right\} \neq \emptyset .
$$

As convexity is preserved for only nonnegative perturbations of nonlinear convex polynomials, we require that $v_{j} \in \mathbb{R}_{+}^{p}, j \in J$. Moreover, it has also been noted in 14 that if this nonnegative restriction of $M$ is dropped, the corresponding robust optimization problem is in general NP-hard, even when $\bar{f}$ and $\bar{g}_{j}$ are all convex quadratic functions.

On the other hand, it is known that in the case where $\bar{f}$ and $\bar{g}_{j}$ are convex quadratic functions, and $M:=\mathbb{B}_{p} \cap \mathbb{R}_{+}^{p}$ (the so-called restricted ellipsoidal uncertainty set), the optimal value of $\left(R P_{\bar{\alpha}}\right)$ can be found by solving a semi-definite programming problem (see 114 and for an extension to a class of convex polynomial programs, see 20]). Various computationally tractable classes of robust counterparts of the form $\left(R P_{\bar{\alpha}}\right)$ in the more general case, where $\bar{f}$ and $\bar{g}_{j}$ are convex polynomials, are also given in 20.

Now, consider the family of robust counterparts of the original problem $(P)$ :

$$
\left(R P_{\bar{\alpha}, \alpha}\right) \quad \min _{x \in \mathbb{R}^{n}}\left\{\bar{f}(x): \bar{g}_{j}(x)+\sum_{l=1}^{p} v_{j}^{l} \bar{g}_{j}^{l}(x)+a_{j}^{T} x+b_{j} \leq 0, \forall\left(v_{j},\left(a_{j}, b_{j}\right)\right) \in\left(\bar{\alpha}_{j}+\alpha_{j}\right)\left(M \times \mathbb{B}_{n+1}\right), j \in J\right\},
$$

where $\alpha_{j} \geq 0, j \in J=\{1,2, \ldots, q\}$ and $\bar{\alpha}=\left(\bar{\alpha}_{1}, \ldots, \bar{\alpha}_{q}\right)$.

Definition 2.1 (Radius of robust feasibility) The radius of robust feasibility, $\rho(\bar{g}, \bar{\alpha})$, associated to $\bar{g}=\left(\bar{g}_{1}, \ldots, \bar{g}_{q}\right)$ and $\bar{\alpha} \geq 0$, is defined to be

$$
\rho(\bar{g}, \bar{\alpha}):=\sup \left\{\min _{j \in J} \alpha_{j}:\left(R P_{\bar{\alpha}, \alpha}\right) \text { is feasible }\right\} .
$$


It is interesting to note that in the case where $\bar{\alpha}=0_{q}$ the radius $\rho(\bar{g}, \bar{\alpha})$ provides the maximal size of the ball uncertainty set under which robust feasibility of $(P)$ is guaranteed.

Recall that an extended real-valued function $h$ on $\mathbb{R}^{n}$ is called proper if $h(x)>-\infty$ for all $x \in \mathbb{R}^{n}$ and there exists $x_{0} \in \mathbb{R}^{n}$ such that $h\left(x_{0}\right)<+\infty$. Denote by $\Gamma\left(\mathbb{R}^{n}\right)$ the class of proper convex lower semicontinuous (lsc) extended real-valued functions. Now let $h \in \Gamma\left(\mathbb{R}^{n}\right)$. The effective domain and the epigraph of $h$ are defined respectively as follows:

$$
\operatorname{dom} h=\left\{x \in \mathbb{R}^{n}: h(x)<+\infty\right\} \text { and epi } h=\left\{(x, \gamma) \in \mathbb{R}^{n} \times \mathbb{R}: x \in \operatorname{dom} h, h(x) \leq \gamma\right\} .
$$

The conjugate function of $h, h^{*}: \mathbb{R}^{n} \rightarrow \mathbb{R} \cup\{+\infty\}$, is defined by $h^{*}(v)=\sup \left\{v^{T} x-h(x):\right.$ $x \in \operatorname{dom} h\}$. Note that, for $h_{1}, h_{2} \in \Gamma\left(\mathbb{R}^{n}\right)$,

$$
\operatorname{epi}\left(h_{1}+h_{2}\right)^{*}=\operatorname{epi} h_{1}^{*}+\operatorname{epi} h_{2}^{*}
$$

provided either $h_{1}$ or $h_{2}$ is a real-valued convex function. Moreover, for a proper lsc convex function $h$, we have

$$
\operatorname{epi}(\alpha h)^{*}=\alpha \operatorname{epi} h^{*}+\left(\left\{0_{n}\right\} \times \mathbb{R}_{+}\right) \text {for } \alpha \geq 0 .
$$

The following result plays a key role in the next section in developing upper bounds for the radius of robust feasibility.

Lemma $2.1(\underline{\underline{\mid 10}})$ Let $h_{t} \in \Gamma\left(\mathbb{R}^{n}\right)$ for all $t \in T$ (an arbitrary index set). Then, $\left\{x \in \mathbb{R}^{n}\right.$ : $\left.h_{t}(x) \leq 0, t \in T\right\} \neq \emptyset$ if and only if $(0,-1) \notin \mathrm{cl}$ cone $\left(\bigcup_{t \in T}\right.$ epi $\left.h_{t}^{*}\right)$.

In order to establish bounds for the radius of robust feasibility, we need the following lemma.

Lemma 2.2 Let $h_{j}: \mathbb{R}^{n} \rightarrow \mathbb{R}, j \in J$, be convex functions. Let $\beta \geq 0$, and let $Z_{0} \subset \mathbb{R}^{n+1}$ be a compact and convex set with $0_{n+1} \in \operatorname{int} Z_{0}$. Suppose that $\left(0_{n},-1\right) \in \operatorname{cl}$ cone $\left(\bigcup_{j \in J}\right.$ epi $\left.h_{j}^{*}+\beta Z_{0}\right)$. Then, for all $\delta>0$, we have $\left(0_{n},-1\right) \in \operatorname{cone}\left(\bigcup_{j \in J}\right.$ epi $\left.h_{j}^{*}+(\beta+\delta) Z_{0}\right)$.

Proof. We proceed by the method of contradiction and assume that there exists $\delta>0$ such that $\left(0_{n},-1\right) \notin$ cone $\left(\bigcup_{j \in J}\right.$ epi $\left.h_{j}^{*}+(\beta+\delta) Z_{0}\right)$. Then, the separation theorem implies that there exists $(\xi, r) \in \mathbb{R}^{n+1} \backslash\left\{0_{n+1}\right\}$ such that for all $(y, s) \in$ cone $\left(\bigcup_{j \in J}\right.$ epi $\left.h_{j}^{*}+(\beta+\delta) Z_{0}\right)$,

$$
-r=\left\langle(\xi, r),\left(0_{n},-1\right)\right\rangle \leq 0 \leq\langle(\xi, r),(y, s)\rangle .
$$

Note that $\left(0_{n},-1\right) \in \operatorname{cl}$ cone $\left(\bigcup_{j \in J}\right.$ epi $\left.h_{j}^{*}+\beta Z_{0}\right)$. As $|J|<+\infty$, by passing to subsequence if necessary, the Carathédory's Theorem implies that there exist $j_{l} \in J, l=1, \ldots, n+2,\left\{\mu_{k}^{j_{l}}\right\} \subset$ $\mathbb{R}_{+},\left\{u_{k}^{j_{l}}\right\} \subset \operatorname{dom} h_{j_{l}}^{*},\left\{\epsilon_{k}^{j_{l}}\right\} \subset \mathbb{R}_{+}$and $\left(z_{k}^{j_{l}}, t_{k}^{j_{l}}\right) \subset Z_{0}$, such that

$$
\left(y_{k}, s_{k}\right):=\sum_{l=1}^{n+2} \mu_{k}^{j_{l}}\left(\left(u_{k}^{j_{l}}, h_{j_{l}}^{*}\left(u_{k}^{j_{l}}\right)+\epsilon_{k}^{j_{l}}\right)+\beta\left(z_{k}^{j_{l}}, t_{k}^{j_{l}}\right)\right) \rightarrow\left(0_{n},-1\right) \text { as } k \rightarrow \infty .
$$

Let $(w, v) \in Z_{0}$ be such that $\langle(\xi, r),(w, v)\rangle \leq-\eta\|(\xi, r)\|$ for some $\eta>0$ (this is possible as $\left.0_{n+1} \in \operatorname{int} Z_{0}\right)$. Then

$$
\begin{aligned}
\left(y_{k}, s_{k}\right)+\delta(w, v) \sum_{l=1}^{n+2} \mu_{k}^{j_{l}} & =\sum_{l=1}^{n+2} \mu_{k}^{j_{l}}\left(\left(u_{k}^{j_{l}}, h_{j_{l}}^{*}\left(u_{k}^{j_{l}}\right)+\epsilon_{k}^{j_{l}}\right)+\beta\left(z_{k}^{j_{l}}, t_{k}^{j_{l}}\right)+\delta(w, v)\right) \\
& \in \operatorname{cone}\left(\bigcup_{j \in J}\left(\operatorname{epi} h_{j}^{*}+(\beta+\delta) Z_{0}\right)\right) .
\end{aligned}
$$


Now, let us claim that there exists $\gamma>0$ such that $\sum_{l=1}^{n+2} \mu_{k}^{j_{l}} \geq \gamma$ for all $k \in \mathbb{N}$. Granting this, (4) and (5) imply that $-r \leq 0 \leq\left\langle(\xi, r),\left(y_{k}, s_{k}\right)+\delta(w, v) \sum_{l=1}^{n+2} \mu_{k}^{j_{l}}\right\rangle \leq\left\langle(\xi, r),\left(y_{k}, s_{k}\right)\right\rangle-\eta \delta \gamma\|(\xi, r)\|$. Note that $\left(y_{k}, s_{k}\right) \rightarrow\left(0_{n},-1\right)$ and $(\xi, r) \neq 0_{n+1}$. Letting $k \rightarrow \infty$, we arrive to a contradiction and hence, the conclusion follows.

We now turn our attention to the claim. Suppose not, then by passing to the subsequence if necessary, we can assume that $\sum_{l=1}^{n+2} \mu_{k}^{j_{l}} \rightarrow 0$ as $k \rightarrow \infty$. Since

$$
\sum_{l=1}^{n+2} \mu_{k}^{j_{l}}\left(\left(u_{k}^{j_{l}}, h_{j_{l}}^{*}\left(u_{k}^{j_{l}}\right)+\epsilon_{k}^{j_{l}}\right)+\beta\left(z_{k}^{j_{l}}, t_{k}^{j_{l}}\right)\right) \rightarrow\left(0_{n},-1\right) \text { as } k \rightarrow \infty,
$$

and $\beta \sum_{l=1}^{n+2} \mu_{k}^{j_{l}}\left(z_{k}^{j_{l}}, t_{k}^{j_{l}}\right) \rightarrow 0_{n}$ thanks to the compactness of $Z_{0}$, it follows that

$$
\sum_{l=1}^{n+2} \mu_{k}^{j_{l}} u_{k}^{j_{l}} \rightarrow 0_{n} \text { and } \sum_{l=1}^{n+2} \mu_{k}^{j_{l}}\left(h_{j_{l}}^{*}\left(u_{k}^{j_{l}}\right)+\epsilon_{k}^{j_{l}}\right) \rightarrow-1 .
$$

Let $x_{0} \in \mathbb{R}^{n}$. By the definition of the conjugate function, we have $h_{j_{l}}^{*}\left(u_{k}^{j_{l}}\right) \geq\left\langle u_{k}^{j_{l}}, x_{0}\right\rangle-h_{j_{l}}\left(x_{0}\right)$. So,

$$
\sum_{l=1}^{n+2} \mu_{k}^{j_{l}}\left(h_{j_{l}}^{*}\left(u_{k}^{j_{l}}\right)+\epsilon_{k}^{j_{l}}\right) \geq \sum_{l=1}^{n+2} \mu_{k}^{j_{l}} h_{j_{l}}^{*}\left(u_{k}^{j_{l}}\right) \geq \sum_{l=1}^{n+2} \mu_{k}^{j_{l}}\left(\left\langle u_{k}^{j_{l}}, x_{0}\right\rangle-h_{j_{l}}\left(x_{0}\right)\right) .
$$

On the other hand, since $\sum_{l=1}^{n+2} \mu_{k}^{j_{l}} \rightarrow 0$ as $k \rightarrow \infty$, we have $\sum_{l=1}^{n+2} \mu_{k}^{j_{l}} h_{j_{l}}\left(x_{0}\right) \rightarrow 0$ as $k \rightarrow \infty$. So, (6) gives us that

$$
\sum_{l=1}^{n+2} \mu_{k}^{j_{l}}\left\langle u_{k}^{j_{l}}, x_{0}\right\rangle-\sum_{l=1}^{n+2} \mu_{k}^{j_{l}} h_{j_{l}}\left(x_{0}\right) \rightarrow 0 \text { and } \sum_{l=1}^{n+2} \mu_{k}^{j_{l}}\left(h_{j_{l}}^{*}\left(u_{k}^{j_{l}}\right)+\epsilon_{k}^{j_{l}}\right) \rightarrow-1 .
$$

Letting $k \rightarrow \infty$ in (7), we arrive to a contradiction, and so, the conclusion follows.

The radius of robust feasibility formula is expressed in terms of the so-called epigraphical set of the constraint system, defined by

$$
E(\bar{g}):=\operatorname{conv}\left\{\bigcup_{j \in J} \operatorname{epi} \bar{g}_{j}^{*}\right\} .
$$

The notion of epigraphical set was inspired by the concept of hypographical set, introduced and studied in [7] within the framework of stability in linear semi-infinite programming.

Define a set $Z_{j} \subset \mathbb{R}^{n+1}$ by $Z_{j}=\mathrm{clconv}\left\{\bigcup_{\left(v_{j}^{1}, \ldots, v_{j}^{p}\right) \in M} \sum_{l=1}^{p} v_{j}^{l} \operatorname{epi}\left(\bar{g}_{j}^{l}\right)^{*}\right\}+\mathbb{B}_{n+1}$. Then, $Z_{j}$ is a closed and convex set with $0_{n+1} \in \operatorname{int} Z_{j}$ as $0_{p} \in M$.

Now, let us present an upper bound for the radius of robust feasibility for a robust convex optimization problem.

Theorem 2.1 (Upper Bounds for radius of robust feasibility) For $\left(R P_{\bar{\alpha}, \alpha}\right)$, let $\rho(\bar{g}, \bar{\alpha})$ be the radius of robust feasibility as given in (1). Let $E(\bar{g})$ be the epigraphical set as given in (8). Then,

$$
0 \leq \rho(\bar{g}, \bar{\alpha}) \leq \min \left\{\max _{j \in J} \phi_{Z_{j}}(-z): z \in E(\bar{g})\right\}-\min _{j \in J}\left\{\bar{\alpha}_{j}\right\}
$$

Proof. Clearly, by definition $\rho(\bar{g}, \bar{\alpha}) \geq 0$. We prove the upper bound. Let $\alpha=\left(\alpha_{1}, \ldots, \alpha_{q}\right) \in \mathbb{R}_{+}^{q}$ be such that $\left(R P_{\bar{\alpha}, \alpha}\right)$ is feasible. Then, we have

$$
\left\{x \in \mathbb{R}^{n}: \bar{g}_{j}(x)+\sum_{l=1}^{p} v_{j}^{l} \bar{g}_{j}^{l}(x)+a_{j}^{T} x+b_{j} \leq 0, \forall\left(v_{j},\left(a_{j}, b_{j}\right)\right) \in\left(\bar{\alpha}_{j}+\alpha_{j}\right)\left(M \times \mathbb{B}_{n+1}\right), j \in J\right\} \neq \emptyset .
$$


Let $\varphi_{j}(x)=\bar{g}_{j}(x)+\sum_{l=1}^{p} v_{j}^{l} \bar{g}_{j}^{l}(x)+a_{j}^{T} x+b_{j}$ for all $x \in \mathbb{R}^{n}$. Then, from (2), (3) and epi $\left(\bar{g}_{j}\right)^{*}+$ $\left\{0_{n}\right\} \times \mathbb{R}_{+}=\operatorname{epi}\left(\bar{g}_{j}\right)^{*}$, we have epi $\varphi_{j}^{*}=\operatorname{epi}\left(\bar{g}_{j}\right)^{*}+\sum_{l=1}^{p} v_{j}^{l} \operatorname{epi}\left(\bar{g}_{j}^{l}\right)^{*}+\left(a_{j},-b_{j}\right)$. Thus, Lemma 2.1 implies that

$$
\left(0_{n},-1\right) \notin \operatorname{cl} \text { cone }\left(\bigcup_{\substack{\left(v_{j}^{1}, \ldots, v_{j}^{p}\right) \in\left(\bar{\alpha}_{j}+\alpha_{j}\right) M,\left(a_{j}, b_{j}\right) \in\left(\bar{\alpha}_{j}+\alpha_{j}\right) \mathbb{B}_{n+1}, j \in J}}\left\{\operatorname{epi}\left(\bar{g}_{j}\right)^{*}+\sum_{l=1}^{p} v_{j}^{l} \operatorname{epi}\left(\bar{g}_{j}^{l}\right)^{*}+\left(a_{j},-b_{j}\right)\right\}\right) .
$$

On the other hand,

$$
\bigcup_{j \in J}\left\{\operatorname{epi}\left(\bar{g}_{j}\right)^{*}+\left(\bar{\alpha}_{j}+\alpha_{j}\right) Z_{j}\right\} \subset \operatorname{cl} \text { cone }\left(\bigcup_{\substack{\left(v_{j}^{1}, \ldots, v_{j}^{p}\right) \in\left(\bar{\alpha}_{j}+\alpha_{j}\right) M,\left(a_{j}, b_{j}\right) \in\left(\bar{\alpha}_{j}+\alpha_{j}\right) \mathbb{B}_{n+1}, j \in J}}\left\{\operatorname{epi}\left(\bar{g}_{j}\right)^{*}+\sum_{l=1}^{p} v_{j}^{l} \operatorname{epi}\left(\bar{g}_{j}^{l}\right)^{*}+\left(a_{j},-b_{j}\right)\right\}\right) .
$$

So,

$$
(0,-1) \notin \operatorname{cl} \text { cone }\left(\bigcup_{j \in J}\left\{\operatorname{epi}\left(\bar{g}_{j}\right)^{*}+\left(\bar{\alpha}_{j}+\alpha_{j}\right) Z_{j}\right\}\right) .
$$

Now, take any $(u, v) \in E(\bar{g})$. Then, by the definition of $E(\bar{g})$ and the Carathéodory Theorem, there exist $\lambda_{k} \geq 0$ with $\sum_{k=1}^{n+2} \lambda_{k}=1, j_{k} \in J, u_{j_{k}} \in \operatorname{dom}\left(\bar{g}_{j_{k}}\right)^{*}$ and $r_{j_{k}} \geq 0, k=1, \ldots, n+2$, such that $(u, v)=\sum_{k=1}^{n+2} \lambda_{k}\left(u_{j_{k}},\left(\bar{g}_{j_{k}}\right)^{*}\left(u_{j_{k}}\right)+r_{j_{k}}\right)$. Let $\epsilon>0$. Noting that $\sum_{k=1}^{n+2} \lambda_{k}=1$, we get that $\left(0_{n},-\epsilon\right)=\sum_{k=1}^{n+2} \lambda_{k}\left(u_{j_{k}}-u,\left(\bar{g}_{j_{k}}\right)^{*}\left(u_{j_{k}}\right)+r_{j_{k}}-v-\epsilon\right)$, and hence $\left(0_{n},-1\right)=\sum_{k=1}^{n+2} \frac{\lambda_{k}}{\epsilon}\left(u_{j_{k}}-u,\left(\bar{g}_{j_{k}}\right)^{*}\left(u_{j_{k}}\right)+r_{j_{k}}-v-\epsilon\right)=\sum_{k=1}^{n+2} \frac{\lambda_{k}}{\epsilon}\left(\left(u_{j_{k}},\left(\bar{g}_{j_{k}}\right)^{*}\left(u_{j_{k}}\right)+r_{j_{k}}\right)+(-u,-v-\epsilon)\right)$.

Note from the definition of $\phi_{Z_{j}}$ that $(-u,-v-\epsilon) \in \phi_{Z_{j}}(-u,-v-\epsilon) Z_{j}$. So,

$$
\left(0_{n},-1\right) \in \text { cone }\left(\bigcup_{j \in J}\left\{\operatorname{epi}^{*}{ }_{j}+\phi_{Z_{j}}(-u,-v-\epsilon) Z_{j}\right\}\right) .
$$

Thus, (9) implies that $\min _{j \in J}\left\{\bar{\alpha}_{j}+\alpha_{j}\right\} \leq \max _{j \in J} \phi_{Z_{j}}(-u,-v-\epsilon)$. Letting $\epsilon \rightarrow 0$ and noting that, for each $j \in J, \phi_{Z_{j}}$ is a continuous convex function and $\min _{j \in J}\left\{\bar{\alpha}_{j}\right\}+\min _{j \in J}\left\{\alpha_{j}\right\} \leq \min _{j \in J}\left\{\bar{\alpha}_{j}+\alpha_{j}\right\}$, we have $\min _{j \in J} \alpha_{j} \leq \max _{j \in J} \phi_{Z_{j}}(-u,-v)-\min _{j \in J}\left\{\bar{\alpha}_{j}\right\}$. Taking infimum over all $(u, v) \in E(\bar{g})$, we have $\min _{j \in J} \alpha_{j} \leq \min \left\{\max _{j \in J} \phi_{Z_{j}}(-u,-v):(u, v) \in E(\bar{g})\right\}-\min _{j \in J}\left\{\bar{\alpha}_{j}\right\}$. Thus the conclusion follows.

Remark 2.1 (i) We note that, in the special case when $\bar{\alpha}=0_{q}$, the above formula of radius of robust feasibility provides a value for the maximal size of the uncertainty set under which the robust feasible set is nonempty, and so, there is no constraint violations. If the parameter $\alpha$ in the above uncertainty models is greater than the corresponding radius of robust feasibility then the probability of violating at least one constraint of the robust counterpart is 1 . In other words, the radius of robust feasibility determines the least conservative decision for a robust decision maker. The reader is referred to [6, 5] for a detailed study on adjusting the level of conservatism of the robust solutions in terms of probabilistic bounds of constraint violations.

(ii) A close inspection of the proof reveals that Theorem 3.1 continues to hold for problems $\left(P_{\alpha}\right)$ where each, $\bar{g}_{j}, j \in J$, is a convex function rather than a convex polynomial. However, as we see in the following section, we require each $\bar{g}_{j}, j \in J$, to be a polynomial in order to derive computable exact formulas for the radius. 


\section{Exact Formulas for Radius of Robust Feasibility}

In this section, we show that, for the robust convex optimization problem with SOS-convex polynomial constraints under affine data uncertainty, we obtain an exact formula for the radius of robust feasibility. More importantly, in this case, the radius of robust feasibility can be expressed in terms of the optimal value of a convex quadratic optimization problem with sums-of-squares constraints, and so, can be efficiently computed by a SDP problem (e.g. [1, 19]).

To do this, we first recall the definitions of sum-of-squares polynomials and SOS-convex polynomials. We say that a real polynomial $f$ is sum-of-squares if there exist real polynomials $f_{j}, j=1, \ldots, r$, such that $f=\sum_{j=1}^{r} f_{j}^{2}$. The set of all sum-of-squares real polynomials in $x$ is denoted by $\Sigma^{2}[x]$. A real polynomial $f$ on $\mathbb{R}^{n}$ is called SOS-convex if $\sigma(x, y):=f(x)-f(y)-$ $\nabla f(y)^{T}(x-y)$ is a sum-of-squares polynomial in $(x, y)$ on $\mathbb{R}^{n} \times \mathbb{R}^{n}$ (see [1, 18]) or, equivalently, if its Hessian matrix $\nabla^{2} f$ is SOS matrix polynomial (i.e., $\nabla^{2} f(x)=H(x) H(x)^{T}$ for some $H(x) \in \mathbb{R}[x]^{n \times r}$, for some $r \in \mathbb{N}(\underline{15}, \underline{20})$. The class of SOS-convex polynomials includes the classes of separable convex polynomials and convex quadratic functions. Clearly, a SOSconvex polynomial is a convex polynomial, but not all convex polynomials are SOS-convex. An interesting feature of the SOS-convexity is that whether a given polynomial is SOS-convex or not can be checked by solving a related SDP problem; while checking the convexity of a polynomial is in general very difficult $\underline{11}$.

For any given convex function $\bar{f}: \mathbb{R}^{n} \rightarrow \mathbb{R}$, we examine the feasibility of the robust counterpart of the convex program $(P)$ under affine data uncertainty, given by

$$
\left(\widetilde{R P} \alpha_{\alpha_{0}, \alpha}\right) \quad \min _{x \in \mathbb{R}^{n}}\left\{\bar{f}(x): \bar{g}_{j}(x)+a_{j}^{T} x+b_{j} \leq 0, \forall\left(a_{j}, b_{j}\right) \in\left(\alpha_{0}+\alpha_{j}\right) \mathbb{B}_{n+1}, j \in J\right\},
$$

where each $\bar{g}_{j}$ is a SOS-convex polynomial, $j \in J:=\{1, \ldots, q\}, \alpha_{0} \geq 0$ and $\alpha=\left(\alpha_{1}, \ldots, \alpha_{q}\right) \in$ $\mathbb{R}_{+}^{q}$. We assume throughout this section that $\left\{x \in \mathbb{R}^{n}: \bar{g}_{j}(x)+a_{j}^{T} x+b_{j} \leq 0, \forall\left(a_{j}, b_{j}\right) \in\right.$ $\left.\alpha_{0} \mathbb{B}_{n+1}, j \in J\right\} \neq \emptyset$.

Recall that the simplex in $\mathbb{R}^{q}$ is denoted by $\Delta_{q}$, and is given by $\Delta_{q}=\left\{\left(\lambda_{1}, \ldots, \lambda_{q}\right): \lambda_{j} \geq 0\right.$ $\forall j \in J$ and $\left.\sum_{j \in J} \lambda_{j}=1\right\}$.

Theorem 3.1 (Calculating the radius of robust feasibility via SDP) For $\left(\widetilde{R P}_{\alpha_{0}, \alpha}\right)$, let $\rho\left(\bar{g}, \alpha_{0}\right)$ be the radius of robust feasibility as given in (1) replacing $\left(R P_{\bar{\alpha}, \alpha}\right)$ by $\left(\widetilde{R P}_{\alpha_{0}, \alpha}\right)$. Then, $\rho\left(\bar{g}, \alpha_{0}\right)=$ $\rho_{0}-\alpha_{0}$, where $\rho_{0}$ is a nonnegative number satisfying

$$
\rho_{0}^{2}=\min _{(w, r) \in \mathbb{R}^{n} \times \mathbb{R}, \lambda \in \Delta_{q}}\left\{\|(w, r)\|^{2}: r-\langle w, \cdot\rangle+\sum_{j \in J} \lambda_{j} \bar{g}_{j} \in \Sigma^{2}[x]\right\} .
$$

Proof. Letting $M=\{0\}$ in Theorem 2.1 gives $Z_{j}=\mathbb{B}_{n+1}$ and $\phi_{Z_{j}}(z)=\|z\|$ for all $z \in \mathbb{R}^{n}$. Then, applying Theorem 2.1 with $\bar{\alpha}_{j}=\alpha_{0}, j=1, \ldots, q$ gives us that $\rho\left(\bar{g}, \alpha_{0}\right) \leq \min \{\|(w, r)\|$ : $(w, r) \in E(\bar{g})\}-\alpha_{0}$, where $E(\bar{g})$ is the epigraphical set defined as in (8). We now show that $\rho\left(\bar{g}, \alpha_{0}\right)=\min \{\|(w, r)\|:(w, r) \in E(\bar{g})\}-\alpha_{0}$. To see this, we proceed by the method of contradiction and suppose that $\rho\left(\bar{g}, \alpha_{0}\right)<\min \{\|(w, r)\|:(w, r) \in E(\bar{g})\}-\alpha_{0}$. Let $\delta>0$ with $\rho\left(\bar{g}, \alpha_{0}\right)+2 \delta<\min \{\|(w, r)\|:(w, r) \in E(\bar{g})\}-\alpha_{0}$. From the definition of $\rho\left(\bar{g}, \alpha_{0}\right)$, we have

$$
\left\{x \in \mathbb{R}^{n}: \bar{g}_{j}(x)+a_{j}^{T} x+b_{j} \leq 0, \forall\left(\left(a_{j}, b_{j}\right)\right) \in\left(\alpha_{0}+\rho\left(\bar{g}, \alpha_{0}\right)+\delta\right) \mathbb{B}_{n+1}, j \in J\right\}=\emptyset .
$$

Thus, Lemma 2.1 implies that

$$
\begin{aligned}
\left(0_{n},-1\right) & \in \operatorname{cl} \text { cone }\left(\bigcup_{\left(a_{j}, b_{j}\right) \in \mathbb{B}_{n+1} j \in J}\left\{\operatorname{epi}\left(\bar{g}_{j}\right)^{*}+\left(\alpha_{0}+\rho\left(\bar{g}, \alpha_{0}\right)+\delta\right)\left(a_{j},-b_{j}\right)\right\}\right) \\
& =\operatorname{cl} \text { cone }\left(\bigcup_{j \in J}\left\{\operatorname{epi}\left(\bar{g}_{j}\right)^{*}+\left(\alpha_{0}+\rho\left(\bar{g}, \alpha_{0}\right)+\delta\right) \mathbb{B}_{n+1}\right\}\right) .
\end{aligned}
$$


Applying Lemma 2.2 to $\bar{g}_{j}, j \in J$, and $\beta=\alpha_{0}+\rho\left(\bar{g}, \alpha_{0}\right)+\delta$, we have

$$
\left(0_{n},-1\right) \in \text { cone }\left(\bigcup_{j \in J} \operatorname{epi}\left(\bar{g}_{j}^{*}\right)+\left(\alpha_{0}+\rho\left(\bar{g}, \alpha_{0}\right)+2 \delta\right) \mathbb{B}_{n+1}\right) .
$$

Then, by the Carathédory Theorem, there exist $\lambda_{l} \geq 0, j_{l} \in J, u_{j_{l}} \in \operatorname{dom}\left(\bar{g}_{j_{l}}\right)^{*}, s_{j_{l}} \geq 0$, and $\left(w_{j l}, r_{j l}\right) \in \mathbb{B}_{n+1}, l=1, \ldots, n+2$, such that

$$
\left(0_{n},-1\right)=\sum_{l=1}^{n+2} \lambda_{l}\left(\left(u_{j_{l}},\left(\bar{g}_{j_{l}}\right)^{*}\left(u_{j_{l}}\right)+s_{j_{l}}\right)+\left(\alpha_{0}+\rho\left(\bar{g}, \alpha_{0}\right)+2 \delta\right)\left(w_{j_{l}}, r_{j_{l}}\right)\right) .
$$

Clearly, $\sum_{l=1}^{n+2} \lambda_{l} \neq 0$. Dividing by $\sum_{l=1}^{n+2} \lambda_{l}$ on both sides and rearranging terms, it follows that $\left(\alpha_{0}+\rho\left(\bar{g}, \alpha_{0}\right)+2 \delta\right) \sum_{l=1}^{n+2} \frac{\lambda_{l}}{\sum_{l=1}^{n+2} \lambda_{l}}\left(-w_{j_{l}},-r_{j_{l}}\right) \in \sum_{l=1}^{n+2} \frac{\lambda_{l}}{\sum_{l=1}^{n+2} \lambda_{l}}\left(u_{j_{l}},\left(\bar{g}_{j_{l}}\right)^{*}\left(u_{j_{l}}\right)+s_{j_{l}}\right)+\frac{1}{\sum_{l=1}^{n+2} \lambda_{l}}\left(0_{n}, 1\right)$.

Let $\left(w^{*}, r^{*}\right):=\sum_{l=1}^{n+2} \frac{\lambda_{l}}{\sum_{l=1}^{n+2} \lambda_{l}}\left(w_{j_{l}}, r_{j_{l}}\right) \in \mathbb{B}_{n+1}$. So, $\left(\alpha_{0}+\rho\left(\bar{g}, \alpha_{0}\right)+2 \delta\right)\left(-w^{*},-r^{*}\right) \in E(\bar{g})$ as $\left(0_{n}, 1\right)$ is a recession direction of $E(\bar{g})$. It then follows that

$$
\begin{aligned}
\min \{\|(w, r)\|:(w, r) \in E(\bar{g})\} \leq\left(\alpha_{0}+\rho\left(\bar{g}, \alpha_{0}\right)+2 \delta\right)\left\|\left(w^{*}, r^{*}\right)\right\| & \leq \alpha_{0}+\rho\left(\bar{g}, \alpha_{0}\right)+2 \delta \\
& <\min \{\|(w, r)\|:(w, r) \in E(\bar{g})\},
\end{aligned}
$$

which is impossible. Therefore, we see that $\rho\left(\bar{g}, \alpha_{0}\right)=\min \{\|(w, r)\|:(w, r) \in E(\bar{g})\}-\alpha_{0}$.

The conclusion will follow if we show the equivalence that $(w, r) \in E(\bar{g})$ if and only if there exist $\lambda \in \Delta_{q}$ such that $r-\langle w, \cdot\rangle+\sum_{j \in J} \lambda_{j} \bar{g}_{j} \in \Sigma^{2}[x]$, because

$$
\rho\left(\bar{g}, \alpha_{0}\right)=\min _{(w, r) \in \mathbb{R}^{n+1}}\{\|(w, r)\|:(w, r) \in E(\bar{g})\}-\alpha_{0}=\rho_{0}-\alpha_{0},
$$

where $\rho_{0}$ is a nonnegative number satisfying $\rho_{0}^{2}=\min _{(w, r) \in \mathbb{R}^{n+1}}\left\{\|(w, r)\|^{2}:(w, r) \in E(\bar{g})\right\}$. Let $(w, r) \in E(\bar{g})$. From the definition of $E(\bar{g})$, there exist $\bar{\lambda}_{l} \geq 0, l=1, \ldots, n+2$ with $\sum_{l=1}^{n+2} \bar{\lambda}_{l}=1$, $j_{l} \in J, u_{j_{l}} \in \operatorname{dom} \bar{g}_{j_{l}}^{*}, r_{j_{l}} \geq 0$ and $s \geq 0$ such that $(w, r)=\sum_{l=1}^{n+2} \bar{\lambda}_{l}\left(u_{j_{l}}, \bar{g}_{j_{l}}^{*}\left(u_{j_{l}}\right)+r_{j_{l}}\right)+\left(0_{n}, s\right)$. This shows that $w=\sum_{l=1}^{n+2} \bar{\lambda}_{l} u_{j_{l}}$ and $r \geq \sum_{l=1}^{n+2} \bar{\lambda}_{l} \bar{g}_{j_{l}}^{*}\left(u_{j_{l}}\right)$. It then follows that, for all $x \in \mathbb{R}^{n}$,

$$
r-\langle w, x\rangle+\sum_{l=1}^{n+2} \bar{\lambda}_{l} \bar{g}_{j_{l}}(x)=r-\sum_{l=1}^{n+2} \bar{\lambda}_{l}\left(\left\langle u_{j_{l}}, x\right\rangle-\bar{g}_{j_{l}}(x)\right) \geq 0
$$

Let $J^{\prime}:=\left\{j_{1}, \ldots, j_{n+2}\right\} \subset J$ and define, for each $j \in J, \lambda_{j}= \begin{cases}\sum_{1 \leq l \leq n+2, j_{l}=j} \bar{\lambda}_{l}, & \text { if } \quad j \in J^{\prime}, \\ 0, & \text { if } \quad j \notin J^{\prime} .\end{cases}$ Then, $\lambda \in \Delta_{q}$ and $h:=r-\langle w, \cdot\rangle+\sum_{j \in J} \lambda_{j} \bar{g}_{j}=r-\langle w, \cdot\rangle+\sum_{l=1}^{n+2} \bar{\lambda}_{l} \bar{g}_{j_{l}}, \quad$ is a SOS-convex polynomial which always takes nonnegative values. Then, the Frank-Wolfe Theorem for convex polynomials implies that $\inf _{x \in \mathbb{R}^{n}} h(x)$ is attained $\left[2\right.$. Let $x_{0} \in \operatorname{argmin}_{x \in \mathbb{R}^{n}} h(x)$. Then, $h\left(x_{0}\right) \geq 0$ and $\nabla h\left(x_{0}\right)=0$. Note that the definition of SOS-convex polynomial implies that

$$
\sigma(x, y):=h(x)-h(y)-\nabla h(y)^{T}(x-y)
$$

is a sum-of-squares polynomial. Letting $y=x_{0}$, we see that $h-h\left(x_{0}\right)=\sigma_{0}$, where $\sigma_{0}$ is a sum-of-squares polynomial in $x$ given by $\sigma_{0}(x)=\sigma(x, 0)$ for all $x \in \mathbb{R}^{n}$. Thus, $h=\sigma_{0}+h\left(x_{0}\right)$ is also a sum-of-squares polynomial.

Conversely, let $(w, r) \in \mathbb{R}^{n} \times \mathbb{R}$ and $\lambda \in \Delta_{q}$ be such that $r-\langle w, \cdot\rangle+\sum_{j \in J} \lambda_{j} \bar{g}_{j} \in \Sigma^{2}[x]$. Then, $r \geq\langle w, x\rangle-\sum_{j \in J} \lambda_{j} \bar{g}_{j}(x)$ for all $x \in \mathbb{R}^{n}$, and hence $(w, r) \in \operatorname{epi}\left(\sum_{j \in J} \lambda_{j} \bar{g}_{j}\right)^{*}$. As each $\bar{g}_{j}$ is a SOS-convex polynomial (and so, continuous and convex), (2) yields epi $\left(\sum_{j \in J} \lambda_{j} \bar{g}_{j}\right)^{*}=$ $\sum_{j \in J} \lambda_{j} \operatorname{epi}\left(\bar{g}_{j}\right)^{*}$. Thus, $(w, r) \in \operatorname{conv}\left\{\bigcup_{j \in J} \operatorname{epi}\left(\bar{g}_{j}\right)^{*}\right\} \subset E(\bar{g})$. Thus, the equivalence follows.

The following example illustrates how the radius of feasibility can be calculated for a robust convex optimization problem with SOS-convex polynomial constraints. 
Example 3.1 Let $\bar{f}: \mathbb{R} \rightarrow \mathbb{R}$ be a given convex function and let $\bar{g}_{i}: \mathbb{R} \rightarrow \mathbb{R}, i=1,2$, be given by $\bar{g}_{1}(x)=x^{4}-1$ and $\bar{g}_{2}(x)=x^{4}+2 x^{2}-2$. Clearly, $\bar{g}_{1}$ and $\bar{g}_{2}$ are SOS-convex polynomials. Consider the following robust convex optimization problem with $S O S$-convex polynomial constraints:

$$
\begin{array}{cl}
\min _{x \in \mathbb{R}} & \bar{f}(x) \\
\text { s.t. } & \left(x^{4}-1\right)+a_{1} x+b_{1} \leq 0, \forall\left(a_{1}, b_{1}\right) \in \alpha_{1} \mathbb{B}_{2} \\
& \left(x^{4}+2 x^{2}-2\right)+a_{2} x+b_{2} \leq 0, \forall\left(a_{2}, b_{2}\right) \in \alpha_{2} \mathbb{B}_{2} .
\end{array}
$$

Then, the preceding theorem with $\alpha_{0}=0$ implies that

$$
(\rho(\bar{g}, 0))^{2}=\min _{(w, r) \in \mathbb{R}^{2},\left(\lambda_{1}, \lambda_{2}\right) \in \Delta_{2}}\left\{\|(w, r)\|^{2}: r-w x+\lambda_{1}\left(x^{4}-1\right)+\lambda_{2}\left(x^{4}+2 x^{2}-2\right) \in \Sigma^{2}[x]\right\} .
$$

Note that $r-w x+\lambda_{1}\left(x^{4}-1\right)+\lambda_{2}\left(x^{4}+2 x^{2}-2\right) \in \Sigma^{2}[x]$ means that there exists a positive semi-definite matrix $W \in S^{3}$ such that

$$
r-w x+\lambda_{1}\left(x^{4}-1\right)+\lambda_{2}\left(x^{4}+2 x^{2}-2\right)=\left(\begin{array}{c}
1 \\
x \\
x^{2}
\end{array}\right)^{T}\left(\begin{array}{ccc}
W_{1} & W_{2} & W_{3} \\
W_{2} & W_{4} & W_{5} \\
W_{3} & W_{5} & W_{6}
\end{array}\right)\left(\begin{array}{c}
1 \\
x \\
x^{2}
\end{array}\right) .
$$

This shows that

$$
W_{1}=r-\lambda_{1}-2 \lambda_{2}, W_{6}=\lambda_{1}+\lambda_{2}=1,2 W_{2}=-w, 2 W_{3}+W_{4}=2 \lambda_{2} \text { and } W_{5}=0 .
$$

So, we have

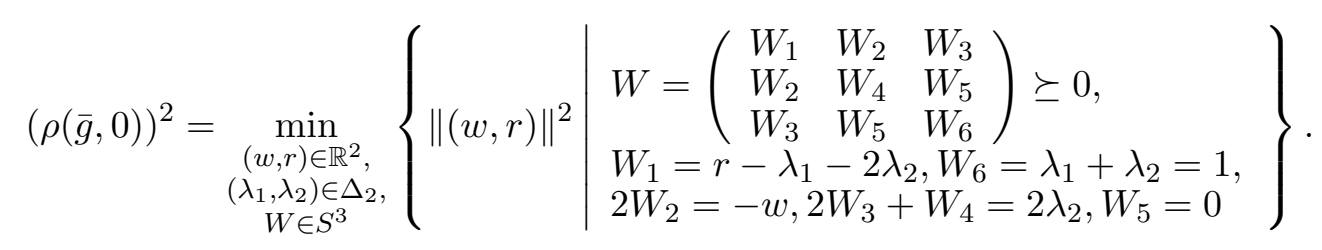

Take any $(w, r) \in \mathbb{R}^{2},\left(\lambda_{1}, \lambda_{2}\right) \in \Delta_{2}$ and $W=\left(\begin{array}{lll}W_{1} & W_{2} & W_{3} \\ W_{2} & W_{4} & W_{5} \\ W_{3} & W_{5} & W_{6}\end{array}\right) \succeq 0$ which satisfies $\left.{ }_{10}\right)$. Then, $r-\lambda_{1}-2 \lambda_{2}=W_{1} \geq 0$, and so, $r \geq \lambda_{1}+2 \lambda_{2} \geq 1$. Thus, $\|(w, r)\|^{2}=w^{2}+r^{2} \geq 1$. So, $\rho(\bar{g}, 0) \geq 1$. On the other hand, letting $\left(\lambda_{1}, \lambda_{2}\right)=(1,0), r=1, w=0$ and $W=\left(\begin{array}{lll}0 & 0 & 0 \\ 0 & 0 & 0 \\ 0 & 0 & 1\end{array}\right) \succeq 0$, we see that $(10)$ holds. So, $\rho(\bar{g}, 0) \leq 1$ and hence $\rho(\bar{g}, 0)=1$.

In fact, converting the optimization problem

$$
\min _{(w, r) \in \mathbb{R}^{2},\left(\lambda_{1}, \lambda_{2}\right) \in \Delta_{2}}\left\{\|(w, r)\|^{2}: r-w x+\lambda_{1}\left(x^{4}-1\right)+\lambda_{2}\left(x^{4}+2 x^{2}-2\right) \in \Sigma^{2}[x]\right\}
$$

into a SDP problem, using the sums-of-squares Matlab toolbox YALMIP [21, 22], and solving it via the SDP numerical software Sedumi, we also obtain the optimal value 1 , and hence $\rho(\bar{g}, 0)=1$.

In the special case of robust optimization problems with convex quadratic constraints we see that the radius of robust feasibility can be calculated via SDP methods.

Corollary 3.1 (Robust convex quadratic optimization) For $\left(\widetilde{R P}_{\alpha_{0}, \alpha}\right)$, let $\bar{g}_{j}(x)=\frac{1}{2} x^{T} A_{j} x+$ $a_{j}^{T} x+\gamma_{j}, j \in J$ be convex quadratic functions with $\left\{x \in \mathbb{R}^{n}: \bar{g}_{j}(x) \leq 0, j \in J\right\} \neq \emptyset$. Let $\rho\left(\bar{g}, \alpha_{0}\right)$ be the radius of robust feasibility as given in (1) replacing $\left(R P_{\bar{\alpha}, \alpha}\right)$ by $\left(\widetilde{R P}_{\alpha_{0}, \alpha}\right)$. Then, $\rho\left(\bar{g}, \alpha_{0}\right)=\sqrt{\mu_{0}}-\alpha_{0}$, where $\mu_{0}$ is a nonnegative number given by

$$
\mu_{0}=\min _{\substack{\mu \in \mathbb{R} \\
(w, r) \in \mathbb{R}^{n} \times \mathbb{R}, \lambda \in \Delta_{q}}}\left\{\mu:\left(\begin{array}{cc}
\sum_{j \in J} \lambda_{j} A_{j} & -w+\sum_{j \in J} \lambda_{j} a_{j} \\
-w+\sum_{j \in J} \lambda_{j} a_{j} & 2\left(r+\sum_{j \in J} \lambda_{j} \gamma_{j}\right)
\end{array}\right) \succeq 0 \text { and }\left(\begin{array}{ccc}
I_{n} & 0 & w \\
0 & 1 & r \\
w^{T} & r & \mu
\end{array}\right) \succeq 0\right\}
$$


Proof. Note that for a quadratic function $f(x)=\frac{1}{2} x^{T} A x+a^{T} x+\gamma, f$ is a sum-of-squares polynomial is equivalent to $f$ always takes nonnegative values, and can be further equivalently rewritten as $\left(\begin{array}{cc}A & a \\ a & 2 \gamma\end{array}\right) \succeq 0$. Thus, the conclusion follows by applying Theorem 3.1 and noting that $\|z\|^{2} \leq s,(z, s) \in \mathbb{R}^{n+1} \times \mathbb{R} \Leftrightarrow\left(\begin{array}{cc}I_{n+1} & z \\ z & s\end{array}\right) \succeq 0$.

The following example illustrates how to find the radius of robust feasibility for a uncertain convex program with convex quadratic constraint using our formula in Corollary 3.1.

Example 3.2 Consider the following 2-dimensional robust convex optimization problem

$(E P) \quad \min _{x \in \mathbb{R}^{2}}\left\{\bar{f}(x):\left(\|x\|^{2}-1\right)+a^{T} x+b \leq 0\right.$ for all $\left.(a, b) \in \alpha \mathbb{B}_{3}\right\}$.

where $\alpha \geq 0, \bar{f}$ is a real-valued convex function on $\mathbb{R}^{2}$ and $\bar{g}_{1}$ is a convex quadratic function on $\mathbb{R}^{2}$ given by $\bar{g}_{1}(x)=\|x\|^{2}-1$ where $x=\left(x_{1}, x_{2}\right)^{T} \in \mathbb{R}^{2}$. The SDP formula for $\rho(\bar{g}, 0)$ in Corollary 3.1 leads to $\rho(\bar{g}, 0)=1$ because

$$
\mu_{0}=\min \left\{\mu:\left(\begin{array}{ccc}
1 & 0 & -w_{1} \\
0 & 1 & -w_{2} \\
-w_{1} & -w_{2} & 2(r-1)
\end{array}\right) \succeq 0,\left(\begin{array}{cccc}
1 & 0 & 0 & w_{1} \\
0 & 1 & 0 & w_{2} \\
0 & 0 & 1 & r \\
w_{1} & w_{2} & r & \mu
\end{array}\right) \succeq 0\right\}=1,
$$

where the last equality follows by noting that the SDP problem takes value 1 with $\left(w_{1}, w_{2}, r, \mu\right)=$ $(0,0,1,1)$ and any feasible point $\left(w_{1}, w_{2}, r, \mu\right)$ of this SDP problem must satisfy $r \geq 1$ and $\mu \geq$ $r^{2} \geq 1$.

In this case, one can also compute the radius of robust feasibility directly using the definition. To see this, note that $\bar{g}(x)+a^{T} x+b \leq 0$ for all $(a, b) \in \alpha \mathbb{B}_{3}$ if and only if $\|x\|^{2}-1+\alpha \sqrt{\|x\|^{2}+1} \leq$ 0 , which is, in turn, equivalent to $\|x\|^{2} \leq \frac{\left(2+\alpha^{2}\right)-\sqrt{8 \alpha^{2}+\alpha^{4}}}{2}$. This shows that

$$
\rho(\bar{g}, 0)=\sup \left\{\alpha: \exists x \text { s.t. }\|x\|^{2} \leq \frac{\left(2+\alpha^{2}\right)-\sqrt{8 \alpha^{2}+\alpha^{4}}}{2}\right\}=1 .
$$

Remark 3.1 (Robust linear optimization) In the special case of a robust linear programming problem with a ball data uncertainty set and $\alpha_{0}=0$, where $\bar{g}_{j}(x)=\bar{a}_{j}^{T} x-\bar{b}_{j}$, the corresponding epigraphical set becomes $E(\bar{g})=\operatorname{conv}\left\{\left(\bar{a}_{j}, \bar{b}_{j}\right): j \in J\right\}+\mathbb{R}_{+}\left\{\left(0_{n}, 1\right)\right\}$. In this case, the proof of Theorem 2.1 leads to a simple formula for the radius of robust feasibility of uncertain linear program, derived in $\left.\underline{\underline{12}, \underline{\underline{13}]}}: \rho\left(\bar{g}, \alpha_{0}\right)=d\left(0_{n}, E(\bar{g})\right)=d\left(0_{n}, \operatorname{conv}\left\{\left(\bar{a}_{j}, \bar{b}_{j}\right): j \in J\right\}+\mathbb{R}_{+}\left\{\left(0_{n}, 1\right)\right\}\right)\right)$.

We finish this section with a remark on how the radius of robust feasibility formula for uncertain convex programs under other commonly used uncertainty sets for robust optimization, such as ellipsoids and polytopes, can be defined and estimated using our approach presented in this paper.

Remark 3.2 (Radius of robust feasibility under polytope and ellipsoid uncertainty sets) The radius of robust feasibility formula can also be defined and estimated for certain other commonly used data uncertainty sets for robust optimization [3, 5]. Indeed, for a convex program $(P)$ under affine data uncertainty with ellipsoid uncertainty sets (see [5, Subsection 2.2] and references therein), one can consider the parametric uncertainty set $\left(\alpha_{0}+\beta_{j}\right)\left(\mathcal{U}_{j} \times\left\{\bar{b}_{j}\right\}\right)$, instead of $\left(\alpha_{0}+\alpha_{j}\right) \mathbb{B}_{n+1}$ in our parametric problems in Section 4 , where $\mathcal{U}_{j}:=\left\{\Delta_{j} u: u \in \mathbb{B}_{n}\right\}, j \in J$ and $\Delta_{j}$ is a positive definite $n \times n$ matrix, $j \in J$. The radius of robust feasibility can then be defined as $\rho_{E}\left(\bar{g}, \alpha_{0}\right):=\sup \left\{\min _{j \in J} \beta_{j}: X_{\beta} \neq \emptyset\right\}$, where $X_{\beta}:=\left\{x \in \mathbb{R}^{n}: \bar{g}_{j}(x)+a_{j}^{T} x+b_{j} \leq 0, \forall\left(a_{j}, b_{j}\right) \in\right.$ $\left.\left(\alpha_{0}+\beta_{j}\right)\left(\mathcal{U}_{j} \times\left\{\bar{b}_{j}\right\}\right), j \in J\right\}$.

Let $\mu:=\max \left\{\left\|\Delta_{j} u\right\|: u \in \mathbb{B}_{n}, j \in J\right\}>0$. Then, $\mathcal{U}_{j} \times\left\{\bar{b}_{j}\right\} \subset \mu \mathbb{B}_{n+1}$ for all $j \in J$. So, if $\left(\alpha_{0}+\beta_{j}\right) \mu-\alpha_{0}<\rho\left(\bar{g}, \alpha_{0}\right)$ then $X_{\beta} \neq \emptyset$. Thus, $\rho_{E}\left(\bar{g}, \alpha_{0}\right) \leq \frac{\rho\left(\bar{g}, \alpha_{0}\right)+\alpha_{0}(1-\mu)}{\mu}$. Similar estimates can 
also be derived for problems $(P)$ with polytope uncertainty sets where the parametric uncertainty set $\left(\alpha_{0}+\alpha_{j}\right) \mathbb{B}_{n+1}$ in Section 4 is replaced by $\left(\alpha_{0}+\gamma_{j}\right) P_{j}, j \in J$, where $P_{j}$ is a given polytope containing $0_{n+1}$.

Future work: It would be of great interest to find numerically tractable formulas for classes of convex programs where uncertainty is present in both affine and non-affine data. We established only estimates for the radius in the case of uncertain convex polynomial constraints by taking into account of non-affine data uncertainty. The issues related to the numerical tractability of the estimates and exact formulas for linear as well as convex programs under other commonly used uncertainty sets for robust optimization, such as ellipsoids and polytopes, will provide interesting topics for further study.

Acknowledgements: The authors are extremely grateful to the referee for the insightful suggestions and valuable comments which have contributed to the final preparation of the paper. The first author would like to thank the University of New South Wales for its support during his stay in November/December 2014. This research was partially supported by the Australian Research Council, Discovery Project DP120100467, the MINECO of Spain and FEDER of EU, Grant MTM2014-59179-C2-1-P.

\section{References}

[1] A.A. Ahmadi and P.A. Parrilo, A complete characterization of the gap between convexity and SOS-convexity, SIAM J. Optim. 23 (2013) 811-833.

[2] E.G. Belousov and D. Klatte, A Frank-Wolfe type theorem for convex polynomial programs, Comp. Optim. \& Appl. 22 (2002) 37-48.

[3] A. Ben-Tal, L. El Ghaoui, A. Nemirovski, Robust optimization, Princeton U.P., Princeton, 2009.

[4] A. Ben-Tal, D. den Hertog, J.P. Vial, Deriving robust counterparts of nonlinear uncertain inequalities, Math. Programming 149A (2015) 265-299.

[5] D. Bertsimas, D.B. Brown, C. Caramanis, Theory and applications of robust optimization, SIAM Rev. 53 (2011) 464-501.

[6] D. Bertsimas, M. Sim, The price of robustness, Oper. Res. 52 (2004) 35-53.

[7] M.J. Cánovas, M.A. López, J. Parra, F.J. Toledo, Distance to ill-posedness and the consistency value of linear semi-infinite inequality systems, Math. Programming 103A (2005) 95-126.

[8] M.J. Cánovas, M.A. López, J. Parra, F.J. Toledo, Distance to ill-posedness for linear inequality systems under block perturbations: convex and infinite-dimensional cases, Optimization 60 (2011) 925-946.

[9] M.J. Cánovas, M.A. López, J. Parra, and M.I.Todorov, Stability and well-posedness in linear semiinfinite programming. SIAM J. Optim. 10 (1999) 82-98.

[10] N. Dinh, M.A. Goberna, M.A. López, From linear to convex systems: Consistency, Farkas Lemma and applications, J. Convex Anal. 13 (2006) 279-290.

[11] L. El Ghaoui, H. Lebret, Robust solutions to least-squares problems with uncertain data, SIAM J. Matrix Anal. Appl. 18 (1997) 1035-1064.

[12] M.A. Goberna, V. Jeyakumar, G. Li, J. Vicente-Pérez, Robust solutions of uncertain multiobjective linear semi-infinite programming, SIAM J. Optim. 24 (2014) 1402-1419.

[13] M.A. Goberna, V. Jeyakumar, G. Li, J. Vicente-Pérez, Robust solutions to multi-objective linear programs with uncertain data, European J. Oper. Res. 242 (2015) 730-743.

[14] D. Goldfarb, and G. Iyengar, Robust convex quadratically constrained programs, Math. Program., 97 (2003), no. 3, Ser. B, 495-515.

[15] J.W. Helton, J.W. Nie, Semidefinite representation of convex sets, Math. Program. 122 (2010) 21-64.

[16] V. Jeyakumar, G. Li, Strong duality in robust convex programming: complete characterizations, SIAM J. Optim. 20 (2010) 3384-3407.

[17] V. Jeyakumar, G. Li, Characterizing robust set containments and solutions of uncertain linear programs without qualifications Oper. Res. Letters 38, 2010, 188-194.

[18] V. Jeyakumar, G. Li, A new class of alternative theorems for SOS-convex inequalities and robust optimization, Applicable Analysis 94 (2015) 56-74.

[19] V. Jeyakumar, G. Li, Exact SDP relaxations for classes of nonlinear semidefinite programming problems, Oper. Res. Letters 40 (2012) 529-536.

[20] V. Jeyakumar, G. Li, J. Vicente-Pérez, Robust SOS-convex polynomial optimization problems: exact SDP relaxations, Optim. Letters 9 (2015) 1-18.

[21] J. Lötberg, Pre- and post-processing sum-of-squares programs in practice, IEEE Trans. Automatic Control 54 (2009) 1007-1011.

[22] J. Löfberg, YALMIP: A Toolbox for Modeling and Optimization in MATLAB. In Proceedings of the CACSD Conference, Taipei, Taiwan, 2004. 